\title{
PROCEEDINGS
}

THE ROYAL
POCIETY $\begin{array}{r}\text { Proc. R. Soc. A (2005) 461, 2911-2921 } \\ \text { doi:10.1098/rspa.2005.1534 } \\ \text { Published online 1 August } 2005\end{array}$

\section{Curvature and topology in smectic-A liquid crystals}

\author{
By Christian D. Santangelo and Randall D. Kamien \\ Department of Physics and Astronomy, University of Pennsylvania, \\ Philadelphia, PA 19104, USA \\ (kamien@physics.upenn.edu)
}

\begin{abstract}
Considerations of rotational invariance in one-dimensionally modulated systems such as smectics-A, necessitate nonlinearities in the free energy. The presence of these nonlinearities is critical for determining the layer configurations around defects. We generalize our recent construction for finding exact minima of an approximate nonlinear free energy to the full, rotationally invariant smectic free energy. Our construction exhibits the detailed connection between mean curvature, Gaussian curvature and layer spacing. For layers without Gaussian curvature, we reduce the Euler-Lagrange equation to an equation governing the evolution of a surface. As an example, we determine the layer profile and free energy of an edge dislocation.
\end{abstract}

Keywords: smectics; nonlinear elasticity; edge dislocations; exact solutions

\section{Introduction}

Phases with one-dimensional broken translational invariance provide a rich phenomenology which includes strong fluctuation effects (Landau 1937; Peierls 1934), anomalous elasticity (Grinstein \& Pelcovits 1981, 1982), and grounds states rife with topological defects (Renn \& Lubensky 1988). As these phases are readily realized in smectic-A liquid crystals, lyotropic lamellae and cholesterics, many of these surprising phenomena can be critically and dramatically verified by experiment. When defects are present, the layers must bend and the resulting curvature is, in general, incompatible with equally spaced layers, even locally (DiDonna \& Kamien 2002; Achard et al. 2005). Except in special geometries (e.g. concentric spheres or cylinders), the problem of determining ideal layer configurations in smectics becomes significantly more complicated than the problem of finding minimal configurations for single surfaces. This is apparent in the smectic blue phases, in which minimal surfaces, which have zero bending energy, cannot simultaneously have equal spacing (DiDonna \& Kamien 2002, 2003). In this article, we develop a manifestly rotationally invariant theory which allows us to study a large class of defect configurations. Using the Bogomol'nyi, Prasad and Sommerfield (BPS) decomposition of the free energy (Bogomol'nyi 1976; Prasad \& Sommerfield 1976), we find first order evolution equations for the layers, akin to those in models of mean curvature flow (Deckelnick \& Dziuk 2003) and which generalize and complete earlier work on approximate theories of smectics (Brener \& Marchenko 1999; Santangelo \& Kamien 2003). 
We describe smectic layers with separation $a_{0}$ as surfaces of constant $\phi(\boldsymbol{x})=z-u(\boldsymbol{x})=a_{0} n$; when $n$ is an integer, these level sets correspond to the $n$th layer. The function $u$ describes the deformations of the surfaces from flat layers with normals in the $\hat{z}$ direction. The layer normal is defined by $\hat{n}=\nabla \phi /|\nabla \phi|$. The free energy has a term from the compression strain, $u_{z z}$, that maintains the layer spacing and a term from the bending strain

$$
F=\frac{1}{2} \int \mathrm{d}^{3} x\left[B u_{z z}^{2}+K_{1}(\nabla \cdot \hat{n})^{2}\right] .
$$

Strictly speaking, in the smectic-A phase, there is another degree of freedom, the nematic director. The difference between the director and the layer normal, however, is a high energy or 'optical' mode. In the following we will ignore this mode and assume that the layer normal and nematic director are locked. In this case, the bending energy $(\nabla \cdot \hat{n})^{2}$ arises from the Frank free energy for the nematic (de Gennes \& Prost 1974)

$$
\left.F_{\text {Frank }}=\frac{1}{2} \int \mathrm{d}^{3} x\left\{K_{1}(\nabla \cdot \hat{n})^{2}+K_{2}[\hat{n} \cdot(\nabla \times \hat{n})]^{2}+K_{3}[(\hat{n} \cdot \nabla) \hat{n})\right]^{2}\right\} .
$$

For surfaces defined as level sets of some phase field $\phi(x, y, z), \hat{n} \cdot(\nabla \times \hat{n})=0$ implying the twist term vanishes identically whenever the surface is nonsingular. Near the core of defects, the director and the layer normal differthough the director develops twist, the layer normal does not. Additionally, we will ignore the bend term as, for small deformations, it is higher order in derivatives along the layer normal than the compression $u_{z z}$. However, to properly treat the elastic strain near the core of deformations with large $u_{z z}$, it is necessary to include this term (Pleiner 1988). We will return to this point later and will propose a way to incorporate the bend. For the moment, however, we set $K_{3}=0$.

When boundaries are present, it is necessary to add the saddle-splay energy

$$
\left.F_{\mathrm{SS}}=K_{24} \int \mathrm{d}^{3} x \nabla \cdot[\hat{n}(\nabla \cdot \hat{n})-(\hat{n} \cdot \nabla) \hat{n})\right] .
$$

As a total derivative, the contribution of this term depends only on the boundary conditions. Since the presence of topological defects implies the presence of boundaries as well, this term can contribute to the core energy of a defect.

Both the splay and saddle-splay energies are naturally understood in terms of curvature invariants of the smectic layers. Defining the principle radii of curvatures on each surface, we see that the splay energy and saddle-splay energy can be written in terms of the mean curvature

$$
H=\frac{1}{2} \nabla \cdot \hat{n}=\frac{1}{2}\left(\frac{1}{r_{1}}+\frac{1}{r_{2}}\right)
$$

and the Gaussian curvature

$$
K=\frac{1}{2} \nabla \cdot[\hat{n}(\nabla \cdot \hat{n})-(\hat{n} \cdot \nabla) \hat{n}]=\frac{1}{r_{1} r_{2}},
$$

respectively (see, for instance, Kamien 2002). 
The elastic strain must vanish when the layers are evenly spaced, i.e. when $|\nabla \phi|=1$, and is a rotationally invariant function of derivatives of $\phi$. Though the form $u_{z z}=(1 / 2)\left[1-(\nabla \phi)^{2}\right]$ arises naturally from a Landau theory for the nematic-smectic transition (Chen \& Lubensky 1976), there are other rotationally invariant functional forms in the same universality class. We will find it most convenient to use (Kléman \& Parodi 1975)

$$
u_{z z}=1-|\nabla \phi| .
$$

Though the magnitude may appear unnatural, note that $|\nabla \phi|=\hat{n} \cdot \nabla \phi$, the normal derivative of $\phi$.

Expanding the compression modulus in powers of $u$, and defining $\nabla_{\perp} \equiv \hat{x} \partial_{x}+\hat{y} \partial_{y}$, we find that

$$
u_{z z} \approx \partial_{z} u-\frac{1}{2}\left(\nabla_{\perp} u\right)^{2}+\mathcal{O}\left(u^{3}\right),
$$

which is the expression for strain considered in by Brener \& Marchenko (1999) and in our prior work (Santangelo \& Kamien 2003). The expansion in powers of $u$ must be treated with care, however, because it destroys the rotational invariance of the free energy. For instance, if one were to approximate $u_{z z}=\partial_{z} u$, the layer deformations due to an edge dislocation are incorrect even very far from the dislocation core (Brener \& Marchenko 1999). This occurs because this approximation measures the distance between layers along the $\hat{z}$-axis rather than the layer normal. Using equation (1.7), however, apparently retains enough rotation invariance to correctly describe the layers around an edge dislocation (Ishikawa \& Lavrentovich 1999), which will follow from our general development.

\section{BPS minima of the full free energy}

Our fully rotationally invariant free energy is

$$
F=\frac{B}{2} \int \mathrm{d}^{3} x\left[(1-|\nabla \phi|)^{2}+\lambda^{2}(\nabla \cdot \hat{n})^{2}\right] .
$$

We may rewrite this as a perfect square plus a cross-term

$$
F=\frac{B}{2} \int \mathrm{d}^{3} x\left[(1-|\nabla \phi|-\lambda \nabla \cdot \hat{n})^{2}+2 \lambda(1-|\nabla \phi|) \nabla \cdot \hat{n}\right] .
$$

We now consider just the cross-term

$$
\frac{F_{\mathrm{CT}}}{B}=\lambda \int \mathrm{d}^{3} x \nabla \cdot \hat{n}-\lambda \int \mathrm{d}^{3} x(\hat{n} \cdot \nabla \phi)(\nabla \cdot \hat{n}) .
$$

The first term on the right-hand side of this equation is a total derivative. The second term is proportional to an integral of the mean curvature

$$
I=2 \int \mathrm{d}^{3} x|\nabla \phi| H=\int \mathrm{d}^{3} x \nabla \phi \cdot[\hat{n}(\nabla \cdot \hat{n})] .
$$


Because $\hat{n}$ is a unit vector, $0=(\hat{n} \cdot \nabla) \hat{n}^{2}=2 \hat{n} \cdot(\hat{n} \cdot \nabla) \hat{n}$ and so

$$
\nabla \phi \cdot[(\hat{n} \cdot \nabla) \hat{n}]=0,
$$

because $\nabla \phi$ is parallel to $\hat{n}$. Adding this to the expression (2.4) we have

$$
I=\int \mathrm{d}^{3} x \nabla \phi \cdot[\hat{n}(\nabla \cdot \hat{n})-(\hat{n} \cdot \nabla) \hat{n}]=-2 \int \mathrm{d}^{3} x \phi K+\int \mathrm{d}^{3} x \nabla \cdot(\phi \psi),
$$

where the final equality follows from integration by parts, $\psi \equiv \hat{n}(\nabla \cdot \hat{n})-(\hat{n} \cdot \nabla) \hat{n}$, and $K=(1 / 2) \nabla \cdot \psi$ is the Gaussian curvature of the layers.

This final identity is reminiscent of the celebrated result of Minkowski (Minkowski 1903) used effectively to study bubble geometries (Hilgenfeldt et al. 2001; Hilgenfeldt 2002). Though the Gaussian curvature is an intrinsic quantity, when considering a series of layers it is hopelessly intertwined with the mean curvature and the layer spacing (DiDonna \& Kamien 2002). To understand this further, we may choose an embedding for the surfaces, $\boldsymbol{X}(x, y, n)$ and coordinates $(x, y, n)$, where $x$ and $y$ are coordinates on each layer and $n$ labels the layer. The embedding satisfies the condition $\phi(\boldsymbol{X})=a_{0} n$. For equally spaced layers, $|\nabla \phi|=1$, and we may change variables

$$
\int \mathrm{d}^{3} x|\nabla \phi| H=\int \mathrm{d} x \mathrm{~d} y \mathrm{~d} n \frac{\mathrm{d} z}{\mathrm{~d} n} H=a_{0} \int \mathrm{d} x \mathrm{~d} y \mathrm{~d} n \sqrt{g_{n}(x, y)} H=a_{0} \int \mathrm{d} n \int \mathrm{d} A H,
$$

where $\sqrt{g_{n}} a_{0}=\mathrm{d} z / \mathrm{d} n$ follows from conservation of volume (DiDonna \& Kamien 2003) and $\mathrm{d} A=\sqrt{g_{n}} \mathrm{~d} x \mathrm{~d} y$ is the surface area element of the $n$th layer. Thus when the layers are evenly spaced, the cross-term is the sum of the integrals of the mean curvature over the surfaces. Using Minkowski's (1903) result ${ }^{1}$, the average mean curvature on each layer is related to an integral of the Gaussian curvature

$$
\int \mathrm{d} n \int \mathrm{d} A H=\int \mathrm{d} n \int \mathrm{d} A \hat{n} \cdot\left(\boldsymbol{X}-\boldsymbol{X}_{0}\right) K .
$$

Since for equally spaced layers, $\boldsymbol{X}=\boldsymbol{X}_{0}+a_{0} n \hat{n}$, we have $\hat{n} \cdot\left(\boldsymbol{X}-\boldsymbol{X}_{0}\right)=a_{0} n=\phi$ and it follows that for evenly spaced layers

$$
\int \mathrm{d}^{3} x|\nabla \phi| H=\int \mathrm{d}^{3} x \phi K,
$$

and so our result generalizes Minkowski's theorem (up to the ambiguity in the sign of $H$ and the sign of $\hat{n}$ ). This form of the theorem allows us to relate the phase field to the principal curvatures of the evolving surfaces.

Returning to the full free energy, we have

$$
F=\frac{B}{2} \int \mathrm{d}^{3} x\left[(1-|\nabla \phi|-\lambda \nabla \cdot \hat{n})^{2}+4 \lambda K \phi+2 \lambda \nabla \cdot(\hat{n}-\phi \psi)\right] .
$$

\footnotetext{
${ }^{1}$ See, in particular, eqns (29) and (30). For a modern formulation and proof, see Flanders (1963).
} 


\section{BPS evolution}

It is now possible to simplify our discussion by considering those configurations for which $K=0$ - in other words, for which each layer is a developable surface. Note that in the case of parallel edge dislocations, this condition is easily satisfied as the layers only bend in one direction. For these configurations, the free energy has the form

$$
F=\frac{B}{2} \int \mathrm{d}^{3} x\left[(1-|\nabla \phi|-\lambda \nabla \cdot \hat{n})^{2}+2 \lambda \nabla \cdot(\hat{n}-\phi \psi)\right],
$$

i.e. the sum of a perfect square and a total derivative. The total derivative depends only on the boundary conditions, and so it is fixed by topological considerations.

Therefore, there exists a class of minima given by setting the perfect square to zero and whose energy is determined entirely from topology, the so-called BPS states. This results in the equation

$$
1-|\nabla \phi|=\lambda \nabla \cdot \hat{n} .
$$

Notice that, as is typical with Bogomol'nyi equations in other contexts, equation (3.2) is of lower order than the full Euler-Lagrange equations. It is an evolution equation for the layers: given one layer, it determines the shape and location of all other layers. Note also that the ambiguity in the sign of $\lambda$ allows us to have two different evolution equations. We have exploited this in our solution for parallel edge dislocations in the approximate nonlinear theory (Santangelo \& Kamien 2003).

In terms of the embedding function $\boldsymbol{X}$, we have the deceptively simple evolution equation (setting $a_{0}=1$ )

$$
\hat{n} \cdot \partial_{n} \boldsymbol{X}=\frac{1}{1-2 \lambda H} .
$$

In Monge gauge, $\boldsymbol{X}=x \hat{x}+y \hat{y}+h(x, y, n) \hat{z}$, this equation becomes

$$
\partial_{n} h(x, y)=\frac{\sqrt{1+\left(\nabla_{\perp} h\right)^{2}}}{[1-2 \lambda H(h)]},
$$

where

$$
H=\nabla_{\perp} \cdot\left[\frac{\nabla_{\perp} h}{\sqrt{1+\left(\nabla_{\perp} h\right)^{2}}}\right] .
$$

By choosing $h(x, y, 0)$ and evolving it with equation (3.4), we have determined the layer profile for an edge dislocation numerically and have plotted it in figure 1. One interesting feature of the evolution is that the layer deformation of the full theory is very close to that from the partially nonlinear theory with free energy considered previously (Brener \& Marchenko 1999)

$$
F \approx \frac{B}{2} \int \mathrm{d}^{3} x\left[\left(\partial_{z} u-\frac{1}{2} \nabla_{\perp} u^{2}\right)^{2}+\lambda^{2}\left(\nabla_{\perp}^{2} u\right)^{2}\right] .
$$




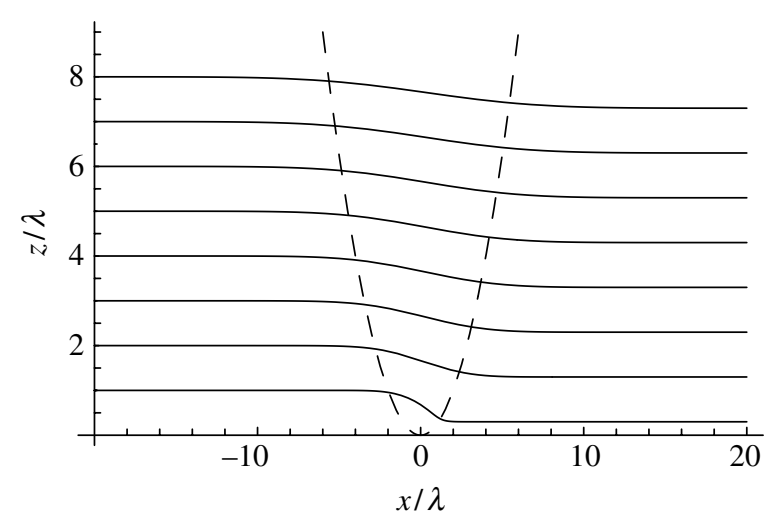

Figure 1. Numerical solution to BPS equation for edge dislocation. Lengths are measured in units of $\lambda$. The dashed curve is the parabola $x^{2}=2 \lambda z$, where the difference between the full evolution (3.2) and the approximate evolution (3.8) is greatest.

To see this, we can consider the long-distance behavior of the BPS evolution, far away from the dislocation core. We choose new dimensionless variables, $\tilde{u}=u / \lambda$, $\alpha=x / \sqrt{\lambda z}$ and $\beta=z / \lambda$. In terms of these new variables, $\lambda \partial_{x}=(1 / \sqrt{\beta}) \partial_{\alpha}$ and $\lambda \partial_{z}=\partial_{\beta}-\alpha /(2 \beta) \partial_{\alpha}$. As $\beta \rightarrow \infty$ for fixed $\alpha$, we may expand the BPS evolution equation in powers of $\beta^{-1}$. To lowest order, we find the equation

$$
\partial_{\beta} \tilde{u}=0,
$$

implying that $\tilde{u}$ depends only on $\alpha$ as $\beta$ becomes large. Therefore, we look for solutions of the form $\tilde{u}(\alpha, \beta)=\tilde{u}_{0}(\alpha)+\tilde{u}_{1}(\alpha) / \beta+\cdots$, order by order in $\beta^{-1}$. Matching terms of order $\beta^{-1}$, we find that $\tilde{u}_{0}$ is determined by

$$
0=\frac{1}{2}\left[\alpha \partial_{\alpha} \tilde{u}_{0}+\left(\partial_{\alpha} \tilde{u}_{0}\right)^{2}\right]+\partial_{\alpha}^{2} u_{0} .
$$

This recapitulates the BPS evolution which follows from (3.6) for edge dislocations, in which the solution is a function only of $\alpha=x / \sqrt{\lambda z}$. Similarly, we could have chosen the variable $\gamma=x / \lambda$ instead of $\beta$ and expanded in powers of $\gamma^{-1}$ for $\alpha$ fixed. For large $\gamma$, we find that

$$
\sqrt{1+\left(\partial_{\gamma} \tilde{u}\right)^{2}}=1+\partial_{\gamma}^{2} \tilde{u}
$$

which implies that $\partial_{\gamma} \tilde{u}=0$ as $\gamma \rightarrow \infty,{ }^{2}$ and the resulting evolution is again given by (3.8). Thus we see that far away from the defect, the nonlinear theory originally considered by Brener and Marchenko captures the essential physics of the problem.

Note that the standard harmonic theory

$$
F_{\text {har }}=\frac{1}{2} \int \mathrm{d}^{3} x\left[B\left(\partial_{z} u\right)^{2}+K_{1}\left(\nabla_{\perp} u\right)^{2}\right]
$$

${ }^{2}$ Let $f=\partial_{\gamma} \tilde{u}$, so $\partial_{\gamma} f=\sqrt{1+f^{2}}-1 \geq 0$. Since, $f \rightarrow 0$ as $\gamma \rightarrow \pm \infty$ for the geometry considered, it follows, since $\partial_{\gamma} f \geq 0$, that $f=0$ for all $\gamma$. 
(a)

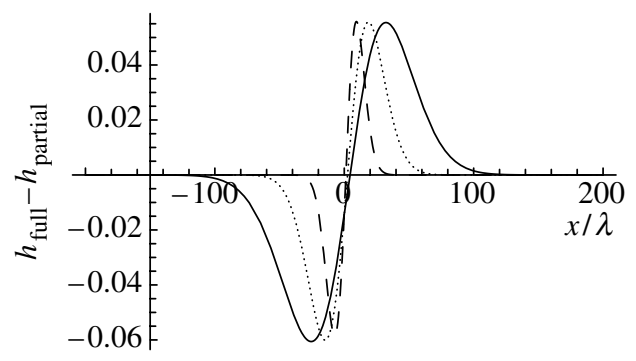

(b)

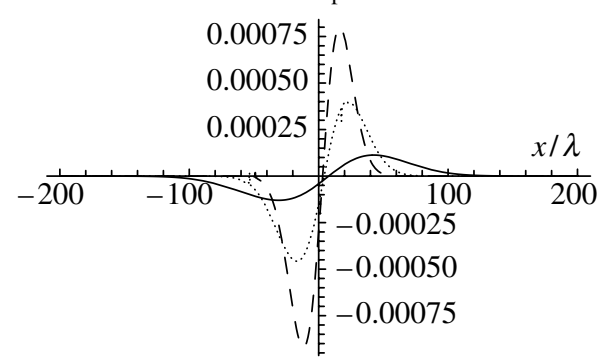

Figure 2. For a single edge dislocation at $x=0$ : $(a)$ Difference between layer height from full BPS theory and height of long wavelength theory for $z=25 \lambda$ (dashed), $z=100 \lambda$ (dotted), and $z=300 \lambda$ (solid), in units of the layer spacing. (b) Plot of $\Gamma_{\text {partial }}=\partial_{z} u-\left(\partial_{x} u\right)^{2} / 2-\lambda \partial_{x}^{2} u$ for $u$ given by the full BPS evolution. As $z$ increases from $z=50 \lambda$ (dashed), $z=100 \lambda$ (dotted) to $z=350 \lambda$ (solid), the full solution approaches the similarity solution of the long distance equation.

is not a consistent approximation to the full nonlinear theory in any sense - as first noted by Grinstein \& Pelcovits $(1981,1982)$, the nonlinear correction to the strain is relevant in the renormalization group sense and, not surprisingly, we find that it is essential even to understand the elasticity of equilibrium configurations. The approximate scaling symmetry $x \rightarrow \Lambda^{1 / 2} x$ and $z \rightarrow \Lambda z$ is responsible for the result that edge dislocations never recover the profile from the quadratic theory along parabolas of constant $\alpha$ (Brener \& Marchenko 1999; Santangelo \& Kamien 2003). Moreover, it suggests that the difference between the full nonlinear theory and the long distance theory will disagree on the parabola $\alpha=$ constant for a defect at the origin - the defect is approximately spread along parabolas for both large $x$ and $z$. This is borne out in our numerical evolution: as shown in figure $2 a$, the difference between the layer height of the full evolution and that given by the long distance theory has peaks which occur on the parabola $x=2 \sqrt{z}$. Figure $2 b$ shows that as $z$ increases, the full solution approaches a solution to the long distance theory, as expected by our analysis.

\section{Further development of BPS evolution}

The Bogomol'nyi equation (3.2) can be rewritten in a quasi-linear form through the Cole-Hopf transformation $S=e^{\phi / \lambda}$

$$
\lambda \nabla \cdot(\hat{n} S)=S
$$

Note that because $\hat{n}=\nabla S /|\nabla S|$, this is a nonlinear equation. However, if $S$ is a solution then so is $c S$ for any consant $c$. This is just a reflection of the underlying global symmetry, $\phi \rightarrow \phi+\tilde{c}$, i.e. the translation symmetry which is spontaneously broken. Expanding (4.1) in powers of $u$ gives a linear equation at both the lowest order (the Euler-Lagrange equation for the harmonic theory) and the next order correction (Santangelo \& Kamien 2003).

Though there is no known analytical solution corresponding to edge dislocations for the full theory, the energy of a dislocation (or multiple dislocations) can be determined simply from the shape of the layers near the 
dislocation core. This is given by the boundary term

$$
F_{\partial}=B \lambda \int_{R} \mathrm{~d}^{3} x[\nabla \cdot \hat{n}-\nabla \cdot(\phi \psi)]=B \lambda \int_{\partial R} \mathrm{~d} A-B \lambda \int_{R} \mathrm{~d}^{3} x \nabla \cdot(\phi \psi),
$$

for some region $R$ with boundary $\partial R$. Since we are considering $K=0$ and configurations for which $\nabla \phi \rightarrow 1$ at infinity, we have $\psi=\nabla \times V$ for some vector $\boldsymbol{V}$. Notice that

$$
\partial_{i}\left(\phi \varepsilon^{i j k} \partial_{j} V_{k}\right)=\partial_{j}\left(\partial_{i} \phi \varepsilon^{i j k} V_{k}\right),
$$

where repeated indices are summed. Thus, the second integral of equation (4.2) gives us the boundary term $\int_{\partial R} \mathrm{~d} A \hat{n}^{i} \partial_{j} \phi V_{k} \varepsilon^{i j k}$ which vanishes identically, again since $\nabla \phi \propto \hat{n}$.

Thus for BPS configurations, the edge dislocation free energy is simply proportional to the area of the layer closest to the core. In particular, suppose the core layer is described by the height function

$$
h(x, 0)=\frac{b}{\sqrt{\pi} \xi} \int_{-\infty}^{x} \mathrm{~d} y \mathrm{e}^{-y^{2} /\left(2 \xi^{2}\right)},
$$

where $\xi$ is the core size. Then we compute the line tension to be

$$
\tau=2 \lambda B \int \mathrm{d} x\left[\sqrt{1+\frac{b^{2}}{\pi \xi^{2}} \mathrm{e}^{-x^{2} / \xi^{2}}}-1\right],
$$

where the first term in brackets is from the surface near the core and the second from the surface at $z \rightarrow \infty$. Expanding in powers of $b^{2}$ recovers the result from equation (3.6) (which agrees with the linear theory), and indeed, the form of the edge dislocation energy is the generalization of the form of the energy found in equation (3.6) (Santangelo \& Kamien 2003). In the opposite limit of very large $b$, however, there is a region of size $\sim \xi \sqrt{\ln \left[b^{2} /\left(\pi \xi^{2}\right)\right]}$ in which the expansion is not valid, and thus for large $b$, the line tension becomes linear in $b$. Note that the split-core model of large $b$ dislocations (Kléman 1983) also exhibits a line tension linear in $b$, but this is a result of an anisotropic core and differs from the phenomenon here. In this limit, one can distinguish between the compression strains $u_{z z}=1-|\nabla \phi|$ and $u_{z z}^{\prime}=\left[1-(\nabla \phi)^{2}\right] / 2$. However, as long as defects are far apart compared to $\xi$, we may compute the energy of several parallel edge dislocations. At long distances, we recover the interactions found from the exact minimization of equation (3.6).

\section{Discussion}

We have discussed the role of curvature and topology in smectic-A liquid crystals, and revealed a close connection between layer spacing, the Gaussian curvature and the mean curvature. For uniformly spaced layers, this connection is related to a celebrated theorem of Minkowski which connects the average mean curvature of a surface to a particular integral of the Gaussian curvature. In the case of smectics with unequal layer spacing, however, we find a generalized 
version of this theorem which is conveniently written in terms of the level set function $\phi$.

When the Gaussian curvature vanishes, we can identify a special class of minima of the full smectic free energy. Our construction is related to the methods used to study field configurations of magnetic monopoles and solitons in field theories (Bogomol'nyi 1976, Prasad \& Sommerfield 1976), vesicle shapes (Benoit, Saxena \& Lookman 2001), and thermal fluctuations (Golubovic \& Wang 1994), and which we have used previously to find minima for an approximate smectic free energy (Santangelo \& Kamien 2003).

It is interesting to note that the variation of the Gaussian curvature term takes a simple form (when holding $\phi$ and $\delta n$ fixed on any boundary)

$$
-\delta\left(\lambda \int \mathrm{d}^{3} x \nabla \phi \cdot \psi\right)=\delta\left(2 \lambda \int \mathrm{d}^{3} x \phi K\right)=2 \lambda \int \mathrm{d}^{3} x K \delta \phi .
$$

This follows from Noether's theorem: since the action on the left is invariant under $\phi \rightarrow \phi+c$, for some constant $c$, the variation of the action results in a conservation law which is identical to the equations of motion. Indeed, this argument holds for the original free energy (2.1) and so the full Euler-Lagrange equation is a conservation law

$$
\nabla \cdot(\hat{n} \Gamma)-\lambda \nabla \cdot\left(\frac{\mathbf{P}^{\mathrm{T}} \cdot \nabla \Gamma}{|\nabla \phi|}\right)=2 \lambda K
$$

where $\Gamma \equiv 1-|\nabla \phi|-\lambda \nabla \cdot \hat{n}$ and $P_{i j}^{\mathrm{T}}=\delta_{i j}-\hat{n}_{i} \hat{n}_{j}$ is the projection operator into the tangent plane of the layers. Note that $\Gamma=0$ is the BPS solution. This sort of conservation law has proven to be useful in understanding focal conic structures and other equilibrium layer configurations (Fournier 1994). One feature that becomes immediately clear is that Gaussian curvature acts as a source for $\Gamma$, and is related to the failure of the BPS construction for surfaces with Gaussian curvature. Even when $K=0$, however, non-zero solutions for $\Gamma$ may exist. In particular, two edge dislocations separated by several layers must be described by a non-zero $\Gamma$. This suggests that superposition fails in this case, even in the computation of the interaction energy.

We might attempt to extend the BPS construction to the case when $K_{3}=K_{1}$. Notice that

$$
\psi^{2}=(\nabla \cdot \hat{n})^{2}+[(\hat{n} \cdot \nabla) \hat{n}]^{2}
$$

which allows us to rewrite the free energy as

$$
F=\frac{B}{2} \int \mathrm{d}^{3} x\left\{[(1-|\nabla \phi|) \hat{n}-\lambda \psi]^{2}+2 \lambda(1-|\nabla \phi| \nabla \cdot \hat{n})\right\} .
$$

The BPS evolution then requires that $\psi \propto \hat{n}$, which implies that these minima have vanishing bend, $(\hat{n} \cdot \nabla) \hat{n}=0$. This implies that the layers are evenly spaced and that $H$ is constant which, in turn, implies that $H$ is 0 (DiDonna \& Kamien 2003). Thus, flat layers are the only exact BPS minima if $K_{3}=K_{1}$. However, it may be possible to generalize our work to allow the director and the layer normal to be independent (though coupled) degrees of freedom. This extension to include the bend energy would require a construction along this avenue. 
Finally, it is possible to repeat the entire BPS construction in a curved background, in analogy with DiDonna \& Kamien (2003), by replacing all derivatives with covariant derivatives, $D_{i}$. It is straightforward to show that the smectic free energy in curved space may be rewritten as

$$
F=\frac{B}{2} \int \mathrm{d} V\left\{\left(1-\sqrt{D_{i} \phi D_{i} \phi}-\lambda D_{i} \hat{n}^{i}\right)^{2}-\lambda \phi\left(2 K-R_{n n}\right)+2 \lambda D_{i} A^{i}\right\}
$$

where $\mathrm{d} V$ is the measure, $\mathrm{d}^{3} x \sqrt{g}$, on the three-dimensional curved space with metric $g_{\alpha \beta}, R_{n n}$ is the normal-normal component of the Ricci tensor in the space, and $A^{i}$ is the result of the integration by parts. Therefore, we find the condition that there exist BPS minima in curved space to be

$$
K=R_{n n} / 2 .
$$

Notice that, in the case of the smectic blue phases, the same condition appeared in order to be able to fill the unit cell volume with equally spaced minimal surfaces (DiDonna \& Kamien 2003). In contrast, Gaussian curvature in flat space necessarily generates mean curvature under normal evolution with equal layer spacing, making such a construction impossible. Similarly, BPS evolution for layers with Gaussian curvature necessarily results in additional mean curvature in flat space. However, this effect may be compensated by the additional curvature of the background space.

To conclude, we have found a large class of exact minima of the full smectic free energy in complete correspondence to the exact minima of equation (3.6). These minima have free energy determined entirely by the boundary conditions and show deviations from the free energy of the same configurations determined from the quadratic free energy approximation. We have applied this theory to edge dislocations and demonstrated that, at long distances, the deformations of the full theory recover those computed from equation (3.6).

The authors would like to acknowledge invaluable discussions with G. Grason. This work was supported by NSF grant DMR01-29804, the donors of the Petroleum Research Fund and a gift from Lawrence J. Bernstein.

\section{References}

Achard, M.-F., Kleman, M., Nastishin, Yu. A. \& Nguyen, H.-T. 2005 Liquid crystal helical ribbons as isometric textures. Eur. Phys. J. E 16, 37-47.

Benoit, J., Saxena, A. \& Lookman, T. 2001 Bogomol'nyi decomposition for vesicles of arbitrary genus. J. Phys. A 34, 9417-9423.

Bogomol'nyi, E. B. 1976 Stability of classical solutions. Sov. J. Nucl. Phys. 24, 449-454.

Brener, E. A. \& Marchenko, V. I. 1999 Nonlinear theory of dislocations in smectic crystals: an exact solution. Phys. Rev. E 59, R4752-R4753.

Chen, J. H. \& Lubensky, T. C. 1976 Landau-Ginzburg mean-field theory for nematic to smectic-C and nematic to smectic-A phase-transitions. Phys. Rev. A 14, 1202-1207.

Deckelnick, K. \& Dziuk, G. 2003 Mean curvature flow and related topics. In Frontiers in numerical analysis (ed. J. F. Blowey, A. W. Craig \& T. Shardlow), pp. 63-108. New York: Springer.

de Gennes, P. G. \& Prost, J. 1974 The physics of liquid crystals. London: Oxford University Press.

DiDonna, B. A. \& Kamien, R. D. 2002 Smectic phases with cubic symmetry: the splay analogue of the blue phase. Phys. Rev. Lett. 89, 215504. 
DiDonna, B. A. \& Kamien, R. D. 2003 Smectic blue phases: layered systems with high intrinsic curvature. Phys. Rev. E 68, 041703.

Flanders, H. 1963 Differential forms, with applications to the physical sciences. New York: Academic Press.

Fournier, J. B. 1994 On the covariant elasticity of smectic-A phases. Eur. J. Phys. 15, 319-324.

Golubović, L. \& Wang, Z.-G. 1994 Kardar-Parisi-Zhang model and anomalous elasticity of twoand three-dimensional smectic-A liquid crystals. Phys. Rev. E 49, 2567-2578.

Grinstein, G. \& Pelcovits, R. A. 1981 Anharmonic effects in bulk smectic liquid-crystals and other one-dimensional solids. Phys. Rev. Lett. 47, 856-859.

Grinstein, G. \& Pelcovits, R. A. 1982 Non-linear elastic theory of smectic liquid-crystals. Phys. Rev. A 26, 915-925.

Hilgenfeldt, S. 2002 Bubble geometry. Nieuw Archief voor Wiskunde 5, 224-230.

Hilgenfeldt, S., Kraynik, A. M., Koehler, S. A. \& Stone, H. A. 2001 An accurate von Neuman's law for three-dimensional foams. Phys. Rev. Lett. 86, 2685-2688.

Ishikawa, T. \& Lavrentovich, O. D. 1999 Dislocation profile in a cholesteric finger texture. Phys. Rev. E 60, R5037-R5039.

Kamien, R. D. 2002 The geometry of soft materials: a primer. Rev. Mod. Phys. 74, 953-971.

Kléman, M. 1983 Points, lines and walls: in liquid crystals, magnetic systems and various ordered media. New York: Wiley.

Kléman, M. \& Parodi, O. 1975 Covariant elasticity for smectics-A. J. Phys. 36, 671.

Landau, L. D. 1937 On the theory of phase transitions II. Phys. Z. Sowjet. 11, 545.

Minkowski, H. 1903 Volumen und Oberfläche. Math. Ann. 57, 447-495.

Peierls, R. E. 1934 Bemerkung ueber Umwandlungstemperaturen. Helv. Phys. Acta Suppl. 7, $81-83$.

Pleiner, H. 1988 Energetics of screw dislocations in smectic-A liquid crystals. Liq. Cryst. 3, 239-258.

Prasad, M. K. \& Sommerfield, C. H. 1976 Solutions of classical gauge field-theories with spin and internal symmetry. Nucl. Phys. B 110, 153-172.

Renn, S. R. \& Lubensky, T. C. 1988 Abrikosov dislocation lattice in a model of the cholesteric to smectic-A transition. Phys. Rev. A 38, 2132-2147.

Santangelo, C. D. \& Kamien, R. D. 2003 Bogomol'nyi-Prasad-Sommerfield configurations in smectics. Phys. Rev. Lett. 91, 045506. 